\section{Thérapeutique des déficits enzymatiques}

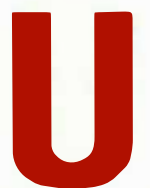

n déficit enzymatique a été trouvé dans au moins deux cents erreurs innées du métabolisme chez l'homme. Est-il possible de remédier à ces déficits? à cette question de nombreuses réponses - toutes incomplètes - ont été apportées.

La plus simple est diététique. La suppression du métabolite toxique permet une vie sensiblement normale au galactosémique, à l'intolérant au fructose, au phénylcétonurique. Dans certains cas, l'introduction massive de vitamines en constitue une : ainsi la pyridoxine à hautes doses empêche les complications de l'homocystinurie chez les déficients en cystathionine-synthase.

Dans la plupart des déficits, il faudrait apporter au malade l'enzyme qui lui fait défaut. Les difficultés sont multiples : quantités considérables requises, réactions immunologiques toujours à craindre et surtout aiguillage correct de l'enzyme vers le tissu atteint. Cette dernière condition explique que les succès les moins discutables, obtenus notamment par Brady, aient porté sur des maladies où la cible principale est le foie, comme la maladie de Gaucher de l'adulte. Les tentatives de prolonger la durée de vie de l'enzyme, ou de mieux l'orienter, en l'insérant dans des structures comme des liposomes ou des hématies hémolysées, puis reconstitutées, ont été dans l'ensemble décevantes.

On s'est donc tourné vers des transplantations, avec l'espoir d'améliorer des résultats encourageants, par exemple ceux des transfusions abondantes dans les déficits immunitaires combinés dus à l'absence d'activité de l'adénosine-desaminase.

Des transplantations ont pu être couronnées de succès lorsqu'il s'agissait de remplacer un organe endommagé par le défaut métabolique. L'exemple typique est la maladie de Fabry : dans ce déficit en $\alpha$-galactosidase lié au sexe, le danger principal est l'insuffisance rénale; une greffe de rein met le malade à l'abri sans le guérir pour autant de sa maladie. On peut également envisager une greffe de foie dans la glycogénose de type I ou la maladie de Wilson par exemple.

Le candidat de choix pour une transplantation est la möelle osseuse, dont les cellules contiennent un arsenal extrêmement vaste d'enzymes. On en a pratiqué, avec des résultats divers, dans des syndromes héréditaires affectant l'érythropoï̀se, le systéme immunitaire, le système réticuloendothélial.

Il est tentant d'appliquer cette méthode aux maladies dues aux déficits en enzymes lysosomiales, tout au moins ceux qui épargnent l'intelligence. Les résultats jusqu'à présent n'entraînent pas tous la conviction. Aussi est-on heureux de signaler un succès remarquable obtenu dans une maladie de surcharge.

La mucopolysaccharidose de type VI ou maladie de Maroteaux-Lamy, due au déficit en arylsulfatase $B$, se manifeste par des anomalies squelettiques, oculaires, cardiaques et pulmonaires sans retard intellectuel. Une fille de 13 ans qui en était atteinte a reçu une transfusion de moëlle osseuse de sa sœur normale. Après un recul de deux ans l'amélioration est considérable : l'activité arylsulfatase $\mathrm{B}$ est normale dans les leucocytes, elle est de $16 \%$ de la normale dans le foie. L'hépatosplénomégalie a régressé, l'acuité visuelle et la mobilité récupérées ont permis la reprise des études.

Un succès comparable a été constaté chez un chat siamois atteint de l'équivalent félin de la maladie. C'est l'occasion de rappeler que les modèles animaux de déficits enzymatiques se sont multipliés au cours des dernières années. Leur étude devrait faire avancer la thérapeutique dans nombre d'affections héréditaires. L'objectif le plus important, dont les difficultés sont évidentes, reste de surmonter l'obstacle de la barrière hémo-méningée.

J.-C. D.

Pyeritz RE. Treatment of inborn errors of metabolism by transplantation. Nature 1984; 312 : 405-6.

Gasper PW, Thrall MA, Wenger DA, et al. Correction of feline arylsulphatase $B$ deficiency (mucopolysaccharidosis VI) by bone marrow transplantation. Nature 1984; $312: 467-9$.

Krivit W, Pierpont ME, Ayaz K, et al. Bone marrow transplantation in the Maroteaux-Lamy syndrome (mucopolysaccharidosis type VI). N Engl 7 Med 20-1 2-1 984; 3 1 1 : 1606-1 1.

\section{Le gène des déficits immunitaires liés au sexe}

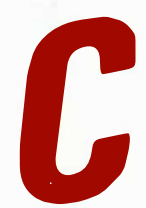

hez l'homme il existe plusieurs types de déficits immunitaires liés au chromosome $X$ et transmis, par conséquent, sur le mode récessif lié au sexe. Certains sont " combinés", intéressant à la fois les cellules $B$ et $T$, d'autres sont localisés au système humoral (atteinte des cellules $B)$, d'autres enfin affectent, outre les $B$ et $T$ lymphocytes, d'autres cellules hématopoiétiques comme les plaquettes (Syndrome de Wiskott Aldrich). Chez la souris, un modèle de ce type d'affection est connu; il s'agit de la mutation xid, avec un déficit de l'immunité humorale et des anomalies des lymphocytes $B$ matures. D.I. Cohen et al. $[I, 2]$ viennent d'isoler un clone d'ADN complémentaire qui semble correspondre $\grave{a}$ un gène, ou l'un des gènes impliqués dans cette mutation, situés sur le chromosome $X$ et exprimés spécifiquement aux stades tardifs de la différen- 
ciation lymphocytaire. Le clone reconnaît en fait une famille de IO-I5 gènes dénommés XLR (X-linked, lymphocyte regulated).

La méthode utilisée pour isoler ce clone a été celle du criblage différentiel : une "banque " d' $A D N$ complémentaire de l'ARN d'une cellule $T$ mature a été criblée avec une sonde spécifique des messagers d'une cellule $T$ "helper" épuisés en les séquences communes avec des lymphocytes $T$ supresseurs. L'un des clones rares ainsi détecté (I molécule d'ARN sur 50000) reconnaît sur l'ADN plusieurs gènes (IO à I5), tous localisés sur le chromosome $X$, et génétiquement liés à la transmission de la mutation xid. Ces gènes, ou certains d'entre eux, sont transmis uniquement aux stades tardif's de la différenciation $B$ lymphocytaire et dans certains lymphocytes $T$. Les $B$ lymphocytes matures de souris xid ne contiennent pas de transcrits des gènes $X L R$, ce qui peut signifier, soit, qu'ils ne sont effectivement pos exprimés du fait d'une mutation les intéressant, soit, que les $B$ lymphocytes normaux synthétisant les $A R N$ messagers $X L R$ sont absents dans la souris xid. Quoiqu'il en soit, la découverte d'une famille génique semblant responsable de tout ou partie du contrôle par le chromosome $X$ de la différenciation du système immunitaire est de nature à faire progresser rapidement notre connaissance tant des syndromes de déficit immunitaire que de la régulation de l'acquisition par les cellules lymphocytaires de la compétence immunologique. Plus pratiquement, la possession de telles sondes d'ADN devrait permettre la mise au point rapide du diagnostic prénatal de ce type d'affection chez l'homme.

A. $\mathbf{K}$.

I. Cohen DI, Hedrick SM, Nielsen EA, et al. Isolation of a cDNA clone corresponding to an $\mathrm{X}$-linked gene family (XLR) closely linked to the murine immunodeficiency disorder xid. Nature $1985 ; 314: 369-72$

2. Cohen DI, Steinberg AD, Paul WE, Davis $\mathrm{MM}$. Expression of an X-linked gene family (XLR) in late-stage $B$ cells and its alteration by the xid mutation. Nature 1985; 31 4:372-4.

$m / s n^{0} 4$ juin-juillet 85

\section{Maladie d'Alzheimer et somatostatine}

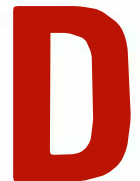

écrite en 1907 par le neurologue allemand Alois Alzheimer, cette maladie frappe une partie notable de la population à partir de 40 ans. Les malades subissent une détérioration intellectuelle progressive les conduisant à la démence en quelques années. Cliniquement difficile à cerner avec précision, l'affection révèle anatomiquement, outre une perte accélérée de neurones, la présence de filaments neurofibrillaires emmêlés (tangles) et de plaques contenant des foyers de débris cellulaires. Un article de R. Wurtman dans le numéro de mars 1985 de Pour la Science passe en revue toutes les interprétations proposées, incluant un modèle génétique (certaines formes sont peut-être familiales), un modèle infectieux inspiré de la maladie de CreutzfeldtJakob, et des troubles circulatoires.

En fait l'ère moderne a été inaugurée par l'apparition depuis 1976 de théories mettant en cause des neurotransmetteurs. Le terrain fut longtemps monopolisé par la théorie cholinergique : il existe une insuffisance de synthèse de l'acétylcholine par suite du déficit en choline acétyltransférase dans le cortex et l'hippocampe chez ces malades. Une atrophie du noyau basal de Meynert, source majeure de l'innervation cholinergique du cortex, peut être mise en évidence.

Mais dans un numéro de Nature de mars 1985 ont paru deux articles qui battent en brèche la primauté du rôle du système cholinergique. C'est désormais la somatostatine $\left({ }^{1}\right)$ qui vient occuper le devant de la scène. La méthode employée utilise avant tout la reconnaissance immunologique de ce peptide dans des coupes de tissu cérébral. On a pu montrer que de nombreux neurones atteints contiennent de la somatostatine et que la destruction de ces neurones est un événement précoce et peut-être critique de la maladie. Au contraire, d'autres neuropeptides ne semblent pas quantitativement altérés dans le cerveau des malades.

Dans la conception de Roberts et al. le déficit cholinergique - dont la réalité n'est aucunement remise en question - pourrait n'être qu'un effet secondaire à la perte de neurones corticaux à somatostatine. L'équipe de Morrison et coll., plus prudente dans ses conclusions, considère que les recherches actuelles tendent surtout à démontrer la multiplicité et la complexité des interventions des neurotransmetteurs dans la pathogénie de la maladie d'Alzheimer.

Un avenir proche devrait fournir de nouvelles précisions sur le rôle des neurotransmetteurs et notamment des neuropeptides. On pourra alors plus efficacement orienter les conceptions pathogéniques, et tenter enfin rationnellement d'enrayer l'évolution, jusqu'à présent inexorable de la maladie.

J.-C. D.

$\left({ }^{1}\right)$ La somatostatine est un peptide présent sous plusieurs formes moléculaires, notamment de 14 et de 28 acides aminés, dérivant d'un précurseur d'une taille d'environ 14000 daltons. Elle a été trouvée dans plusieurs tissus et est abondante dans le système nerveux central.

Roberts GW, Crow T J, Polak JM. Location of neuronal tangles in somatostatin neurones in Alzheimer's disease. Nature $1985 ; 314: 92-4$

Morrison JH, Rogers J, Scherr S, Benoit R, Bloom FE. Somatostatin immunoreactivity in neurotic pla ques of Alzheimer's patients. Nature $1985 ; 314: 90-2$.
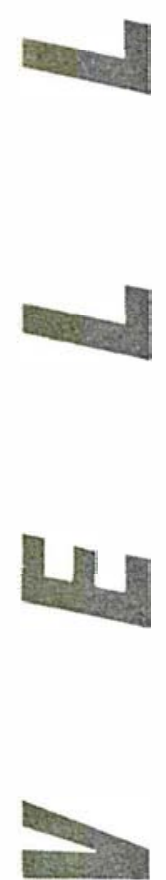

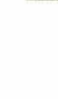

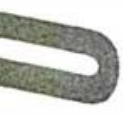

. 